University of Wollongong

Research Online

Faculty of Informatics - Papers (Archive)

Faculty of Engineering and Information

Sciences

21-6-2006

\title{
The Challenges for the Adoption of M-Health
}

Ping Yu

University of Wollongong, ping@uow.edu.au

M. X. Wu

Central Queensland University

H. Yu

Chongqing Institute of Technology, China

G. C. Xiao

Southwest University, Chongqing, China

Follow this and additional works at: https://ro.uow.edu.au/infopapers

Part of the Physical Sciences and Mathematics Commons

\section{Recommended Citation}

Yu, Ping; Wu, M. X.; Yu, H.; and Xiao, G. C.: The Challenges for the Adoption of M-Health 2006.

https://ro.uow.edu.au/infopapers/495

Research Online is the open access institutional repository for the University of Wollongong. For further information contact the UOW Library: research-pubs@uow.edu.au 


\title{
The Challenges for the Adoption of M-Health
}

\author{
Abstract \\ The potential benefit of mobile and wireless information technology for healthcare service delivery, \\ improving patient safety and reducing cost is increasingly recognized and emphasized. To contributing to \\ the knowledge about critical factors determining the success of mobile health ( $\mathrm{m}$-Health) solutions, this \\ study researched the driving force for the advancement of $m$-health solutions. The current advancement \\ of $\mathrm{m}$-health was reviewed. The challenges for developing and deploying $\mathrm{m}$-health applications were \\ addressed. The paper concluded in suggesting the most critical factor for the success of $m$-health. The \\ method used for the study was literature research, market scan and analysis.

\section{Disciplines} \\ Physical Sciences and Mathematics \\ Publication Details \\ This paper was originally published as: Yu, P, Wu, MX, Yu, H \& Xiao, GQ, The Challenges for the Adoption of \\ M-Health, 2006 IEEE International Conference on Service Operations and Logistics and Informatics (SOLI \\ 2006), Shanghai, China, June 21-23 2006, 181-186. Copyright IEEE 2006.
}




\title{
The Challenges for The Adoption of M-Health
}

\author{
Ping Yu, Ming X. Wu, Hui Yu, and Guo Q. Xiao
}

\begin{abstract}
The potential benefit of mobile and wireless information technology for healthcare service delivery, improving patient safety and reducing cost is increasingly recognized and emphasized. To contributing to the knowledge about critical factors determining the success of mobile health (m-Health) solutions, this study researched the driving force for the advancement of m-health solutions. The current advancement of m-health was reviewed. The challenges for developing and deploying m-health applications were addressed. The paper concluded in suggesting the most critical factor for the success of m-health. The method used for the study was literature research, market scan and analysis.
\end{abstract}

Index Terms - Adoption, challenges, CSF, mobile health

\section{INTRODUCTION}

$\mathrm{T}$ He penetration of ubiquitous computing into healthcare domain has seen the growing demand for nationwide Electronic Health Record (EHR) in the major developed countries such as Australia, Canada, England, and USA. There is a continuous demand to link hospital inpatient care to outpatient and long-term care to achieve continuity of care. This is essential for improving patient safety, healthcare quality and reducing cost. Healthcare IT application vendors are working hard towards satisfying healthcare workers' needs for an enabling IT solution to facilitate their providing of right care to the right patient anytime anyplace. Healthcare industry also realizes the great potential pervasive computing could provide to revolutionize the traditional model of healthcare delivery.

There is considerable interest in both vendor and health care provider communities in developing and implementing mobile and wireless healthcare IT applications. However, many system development and implementation issues have to be thoroughly understood and continuously investigated for the successful introduction of mobile health (m-health) solutions into healthcare. The issues include healthcare workers' information needs, workflow and usability requirements, the available technology options and how technology can be best developed and implemented that really suits health care workers' information needs and work flow.

Ping Yu is with Health Informatics Research Center, School of IT and Computer Science, The University of Wollongong, NSW, Australia 2522 (Tel: 0061-2-42215412; Fax: 0061-2-42214170; e-mail: ping@uow.edu.au).

Ming X. Wu is with Faculty of Business and Informatics, Central Queensland University, Australia (e-mail: Robert_wumx@hotmail.com \&wum@syd.cqu.edu.au).

Hui Yu is with School of Biomedical Science, Chongqing Institute of Technology, Chongqing, P.R.China 400050 (e-mail: colinhuiyu@yahoo.com).

Guo Q. Xiao is with Faculty of Computer \& Information Science, Southwest University, Chongqing, P.R.China 400715 (e-mail: gqxiao@swu.edu.cn).
It then discusses the driving forces for the advancement of m-health. The current status of m-health will be reviewed. Afterwards the focus will be given to challenges for the development and deployment of $\mathrm{m}$-health applications. The paper will conclude by briefly stating the critical success factors (CSFs) for the advancement of m-health.

\section{THE DEFINITION OF M-HEALTH}

Mobile Healthcare Alliance refers mobile healthcare as activities and systems that allow healthcare to be provided at the point-of-care, such as patient's beds, wards and homes [1]. This paper believes that m-health refers to healthcare facilitated by the convergence of mobile and desktop healthcare information systems, wireless technology and other networks such as Bluetooth and cellular network, which is composed of people and health care processes that are facilitated by wireless and possibly wired connectivity, desktop and mobile healthcare applications.

The goal of m-health is to utilize health care application written on mobile devices that are connected through the wireless networking and communication technology to improve healthcare safety and outcomes, whereas reducing costs.

\section{A. The Driving Force For The Advancement of M-Health}

The fundamental driving force for any effort in technology innovation in healthcare is complying with regulatory requirements for operation and benefit gains. Besides the general healthcare needs for information technology, such as increasing productivity, capacity and patient service, the particular driving force for the advancement of m-Health is clinician's needs for providing the right care to the right patient at any time in any place. Malkary \& Farrell stated, "Mobile computing does provide an opportunity to improve safety and increase efficiency and productivity at the point of care."'[2].

The implementation of wireless technology is driven by the desire of reducing cost and increasing productivity. Person reported a revenue increase of $5 \%$ to $20 \%$ when doctors bill patient on the move using PatientKeeper software on Personal Digital Assistants (PDAs) at the University of Kansas Medical Center [3]. Shabot commented that return on investment (ROI) for wireless network is typically shorter than one year, which is very attractive for hospital administrators who are burdened by the shortage of budget all the time [4].

Complying with the requirements of Joint Commission on Accreditation of Healthcare Organization (JCAHO) of "providing the right care to the right patient" and reducing medical errors is the driving force for the 
advancement of bar code and radio frequency identification (RFID) technology in USA [5].

According to a report conducted by Global Information, Inc. [7], the four key healthcare area that can be dramatically changed by the introduction of mobile technology are (1) direct patient communication and telemedicine; (2) home healthcare delivery; (3) hospitals and (4) emergency situations. The major benefits of m-health are to facilitate as follows [6]:

- Clinicians to access results, reports and patient data from anywhere.

- Instantaneous inventory tracking to enable a mobile staff to identify what they need on the move.

- A patient's vitals and location be monitored with a simple wristband, which provides better control with improved flexibility over traditional methods.

\section{B. The Current Advancement Of M-Health}

Nowadays public places in major cities in USA have gone wireless with Wi-Fi network integrated with cellular networks, 2G and $3 \mathrm{G}$ networks. The successful integration of WiFi Network with cellular access points enabled a community hospital, Central DuPage Hospital in Winfield, Illinois, USA to facilitate "doctor care and patient convenience" [8]. Physicians can use various forms of electronic equipment, such as cellular phone, PDA phone, pocket PC, Palm and laptop, to enter and access information and communicate with care teams. It marks a new era of advancement in m-health.

According to April 28, 2005 e-newsletter published by Mobile Health Data [9], "There's been an explosion of activity involving hand-held technologies in health care" in USA, "About one third practicing physicians are carrying PDAs. Hundreds of hospitals and clinics are implementing wireless networks and arming clinicians with hand-held computers.".

\section{The Integration of Wireless With Cellular Network}

According to Thompson [10], the WiFi design in Central DuPage Hospital includes wireless access points to split off virtual Local Area Networks (VLANs) that serve multiple wireless populations: clinical systems, voice-enabled Internet phones and clinician hot spot for PDA or laptop access. The cellular access point is scattered throughout the hospital. The cellular network integrated with wireless network provides the opportunity for clinicians to access patient information and communicate with care team anywhere anytime via cell phones or PDA phones. This provides the opportunity for clinicians to access patient's EHR anywhere anytime via Internet based Web Portal.

\section{The Diversity Of Mobile Devices}

The mobile devices on the current market include PDAs, tablet PCs, laptops, smart cell phones and computing devices carried on mobile carts. There is a trend to converge PDA, cell phone, bar code reader with the ability to read RFID coded information into one device. Mobile carts are used for nursing documentation at the point of care. Spyglass Consulting Group's survey found that outpatient and hospital physicians have totally different preferences for mobile health devices [11].

\section{E. The Applications}

Current m-health applications available in the market include applications for emergency, ambulatory and hospital care, clinical nursing, pre-hospital emergency, medical calculation, homecare and personal healthcare.

The functionality includes various alerts for decision support and critical value, reference material such as drug database and allergy information, wireless office system, patient information, scheduling, bedside laboratory material collection and results consultation, blood transmission, e-prescribing and order entry, medication administration, voice dictation and transcription, expensive equipment tracking, communication, clinical trials, charge capture, senses, vital signs and EHR. Bar code technology is popular for patient identification and medication management [2], [4], [5], [10].

\section{THE CHALLENGES FOR M-HEALTH SOLUTIONS}

The most CSFs for m-health solutions are clinician's acceptance and adoption of the solutions. To gain clinicians' acceptance, the whole solution, including network, hardware and software applications, should be fully integrated into clinician's workflow and add value to patient care, administrative convenience and facilitating communication among multi-disciplinary care teams. Most importantly, it relies on a strategically planned, systematically conducted implementation that is fully coordinated, managing change of work culture associated with technology implementation and supportive of end user learning the new practice.

\section{A. People}

Lack of health informatics experts who can bridge the gulf between health and technology significantly hinders the advancement of m-Health. Many hospitals simply do not know what wireless LAN could help health care [12]. On the other hand, most of the experienced physicians are not young. The average age of registered nurses in USA is 48 years old [2]. In general, old people are slower than young ones to accept the new technologies and knowledge. They have barriers to accept the new format of IT-based information management [4]. Therefore, a strong training and education program is critical for smoothing clinicians' learning curve of the new practice.

1) Clinicians' Work Habit And The Kinds of Mobile Devices That Best Match Their Needs

Clinicians' work habit and the kinds of mobile and wireless devices that best match their needs become a critical factor determining success or failure of mobile solution adoption [2], [4], [5], [13]. Physicians wish to use a computing device that can be personalized to their personal preference and individual workflow. Ideally the device would provide the information they need anytime across all the locations where they practice [14]. Johnson and Trakowski mentioned that PDA is the most mobile 
device and appears to gain favor from inpatient clinicians [5]. Spyglass Consulting Group's study found that only $6 \%$ to $9 \%$ of nurses use a PDA [11]. Of these users, $93 \%$ use PDAs for reference applications.

Laptop computers are favored by outpatient physicians [2]. Laptop on mobile cart will be a solution for inpatient documentation providing the problem of difficulty to getting it into patient's room is solved.

Enabling multiple devices is a critical factor for gaining physician acceptance of the new technology. The diversity of devices and choices continuously challenges the IT policy regarding the management of devices.

\section{2) Convenience Of Patients}

The driving force for technology is also convenience of patients and visitors. It would add value to patient care if patients can access their own health care information and educational programs through hotel-type TV channels in front of their beds and visitors can access information and communicate anywhere in the hospital [8].

\section{B. Process}

The CSF for the adoption of m-health solutions is cost saving, seamless integration of applications into workflow and managing cultural change associated with the implementation of $\mathrm{m}$-health solutions.

\section{1) Challenges For Building A Compelling Business Case And Measuring ROI For M-Health}

The application of any technology has to be justified by its cost. There are two different opinions regarding the development of business case for $\mathrm{m}$-health. Some people believed that business cases for mobile computing applications were relatively easy to build because of the high cost of clinical services [15]. Another opinion is that hospitals and health insurance companies, as the major enterprise customers in the health care market, have underinvested in information technologies. Only when the benefit can offset the cost, will the technology be widely adopted into hospital environment.

Although a health care organization may have recognized the need to upgrade their information systems, they are scared of the high upfront cost because the slim profit margin is never enough for the competing business needs [6]. The battle between adopting the new IT technology to improve health care outcomes and the investment pressure will never end.

The common recognized difficulty in measuring ROI for $\mathrm{m}$-health project is one important reason attributes for its low adoption rate. Although costs and cost items are not too difficult to be projected and measured, it is very difficult to estimate benefit items and their values because benefits are often intangible. Intangible items and values are the most difficult to be measured [12].

\section{2) Integration Into Clinician's Workflow}

Interactivity is critical for physician acceptance of the solution. The success of mobile computing depends on how well the solution is integrated with mobile clinicians' workflow, if it fits with the ergonomic needs of busy clinicians and handles the information needs of clinicians at point of care.

\section{3) Managing Cultural Change Associated With Technology Adoption}

As implementing any other technology, understanding the social and political forces underlying technology diffusion is critical for effective management of mobile application implementation process. Enough consultation with end users should be taken on how the application will impact on practice. This will increase acceptance by end users. The vision has to be circulated to the executive management, then end users, that is, everyone to be impacted on the implementation. Ensuring executive support is also critical for the success of the implementation practice [5].

\section{4) Managing Change Faced By Hospital IT Department}

There is a need for IT department in hospital to distribute EHR ubiquitously to clinicians and patients simultaneously. It is also important to manage heterogeneous populations requiring a variety of devices [10]. The model used by Central DuPage Hospital and Harvard Medical School is to let clinicians to select their own devices, whereas the role of IT department is to support clinicians' choice [10].

\section{5) The Application Development Process}

To balance in-house development with commercial off-the-shelf solution is critical for the success of application development. There is a tendency in hospitals in USA to collaborate with vendors to build from the existing vendor products [10], [13]. This effectively avoids wasting resources in reinventing the wheel. It leverages the development experience and expertise of vendors and effectively accommodates a particular organization's needs for mobile technology.

In order to really understand the information and ergonomic needs of clinicians and integrate the requirements effectively into application, more clinicians should be involved into design team. Effort should be focused on how to better translate the requirements into system design [4].

\section{Technology}

1) Coverage Of Networks Including Wifi, Bluetooth And Cellular Network

To accommodate the communication needs for data captured by applications on various medical devices, there is a trend for the convergence of various types of networks including wired connection, wireless connection through WiFi (IEEE802.11 a, b, g), Bluetooth (IEEE802.15.1), cellular network and the emerging 2G and $3 \mathrm{G}$ networks [17]. Although there are possible problems of collision and channel interference, these problems can be solved through careful plan and calculation [17].

\section{2) Applications}

The key features for m-health applications include an integrated user interface, context switching between 
inter- and intra-applications, application interoperability, user defined clinical data categories, decision support, encryption, enterprise security, connectivity and centralized administration [18], [19].

Eighty seven percent participants in the Health Data Management 2005 CIO Survey believe that it is important to link medical devices - including bedside monitors, IV pumps and ventilators to clinical information systems [9]. The ability to add patient picture on wristband has been seen as an additional mechanism to support patient identification [5].

\section{3) Web-Based, Portal Technology}

Web-based, portal based approach has shown its advantage in supporting anywhere access to information that is independent of devices. Instead of managing multiple applications, Web portal gives the convenience of bringing everything under one portal for IT management. Web-based portal technology also provides the advantage of reducing the application complexity at client side and satisfying clinicians' requirement for one interface. Therefore, Web-based portal application appears to be the direction of medical software applications [10].

\section{4) One Vendor Software Product Or Best-Of-Bread} Solution

According to CIO survey conducted by Health Data Management in USA, $65 \%$ of healthcare provider organizations in USA like to rely on one software vendor to provide clinical information systems if there is an acceptable level of satisfaction with the major clinical modules the vendor provides [20]. One CIO of a hospital commented "integration is always a challenge and getting a critical mass of valued data into the hands of the clinician is important for the success of the electronic medical record." [21].

\section{5) RFID And Bar Code Technology}

RFID technology is gaining considerable attention recently as the leading edge auto-identification technology. Some RFID also has write capabilities [5], [24]. A US company Applied Digital, through its subsidiary VeriChip Corp., now will offer implantable and external RFID chips and applications. This includes an infant security system for hospitals, a tracking application for long-term care facilities and an application designed to help health care organizations track supplies and medical equipment [25]. California-based Patient Care Technology Systems has badges using RFID technology to enable health care organizations to track patients and assets across emergency departments [25]. A marketing expert commented, "We think RFID will be what sticks." [23].

There is a trend to imbed wireless RFID and bar code technologies into PDA to offer a real mobile point of care information technology solution. According to the report of Health Data Management [23], Sun Microsystems Inc. "supports RFID and bar code technology, but believes bar coding is an interim solution and that the flexibility of RFID technology makes it the right choice for the future".

\section{Mobile Devices Currently Available In The Market}

The mobile devices currently available in the market include PDAs, smart phones, tablet PCs, laptops, bar code, RFID, mobile clinical carts that integrate a full size laptop or computer with drug dispensing facilities. However, "the right device depends on environment, complexity, personal preference and tasks" [22].

\section{1) PDAs}

There are five major problems associated with PDA-based approach: (1) Small screen size causes resistance to adoption from clinicians. Farrell commented that the average age of nurses in USA is 48 years [2]. Nurses complain that they cannot see what is on the small screen of PDA. (2) The limited data entry options to form-based approach, which is lack of flexibility in handling text-based documentation. It is considered to be the mobile device used for simple tasks, but not for entering complex clinical data due to the limitation of screens [13], [22]. (3) Durability of the devices. Users worry about breaking the device, particularly nurses in long-term care environment where close contact with patients, some of them suffer from dementia, is unavoidable. (4) The device is easily lost or stolen. (5) The limited wireless bandwidth and memory are also problems identified [9].

\section{2) Tablet $P C$}

Tablet PC appears to lose ground at bedside computing [2], [5], [16]. The complaints about tablet PC [2] are (1) too fragile and heavy for carrying around during the period of a shift; (2) too large; (3) limited battery life (normally 3.5 hours) and high cost. (4) Tablet PC does not integrate well with ergonomics in the hospital. However, tablet PC are gaining popularity in outpatient physicians for entering or retrieving patient's EMR at the point of care [11], [22]. Outpatient physicians are twice likely to use a tablet PC than their hospital-based counterparts [22]. Over three-quarters of physicians surveyed thought that tablet PC is suitable for daily clinical use [25]. It has been anticipated that tablet PCs may be taken over by laptops with decreasing size and the option of providing both handwriting and typing functions [16].

\section{3) Mobile Clinical Cart}

Mobile clinical cart provides the advantage of familiar look and feel with desktop application because of its full-screen that is identical to desktop computer and the keyboard for data entry. With laptop fixed on cart, it also avoids the problem of lost or stolen computer. The problem of mobile clinical cart is that older nurses find it is heavy and difficult to manipulate. Some cart is too big and cannot be pushed into patient's room [2]. The design of mobile cart is important for the convenience of usage.

\section{4) Podcast on iPod}

On April 28, 2005, Health Data Management reported that health care CIOs and staffs in IT industry have shown considerable interest to the emerging audio software 
podcast stored on iPOD [26]. The emerging new service podcasts have potential role in medical education and asynchronous online course delivery. It will facilitate student learning through listening to the audio recording of lectures anywhere anytime at the student's convenience.

\section{5) Battery Life}

Battery life is a critical issue for the adoption of mobile health applications. Clinicians require a device that is functional during the full duration of their work shift. Battery life for the current model of tablet or laptop only runs for three and a half hours in the best scenario, which is ergonomically difficult to satisfy the documentation needs of clinicians [2], [9].

\section{E. Computing Standards For M-Health}

It is the critical mission for health informatics community to build the worldwide healthcare computing standards early. Without standards, interoperability between systems will never to be achieved.

Even in USA, there are still no national standards for a clinical vocabulary or standardized data formats [27]. However, the situation will be improved with the EMR standard currently being developed by Health Level Seven (HL7).

\section{F. Security And Privacy Consideration}

Fully conforming to HIPAA guidelines is the regulatory requirement for any health information management in USA. Any health information systems developed have to comply with HIPAA requirement.

Advanced Encryption Standard (AES) has been specified by the National Institute of Standards and Technology in USA as the next generation US government standard for generation of symmetric keys. It will replace the old DES standards. Sutherland commented that Elliptical Curve Cryptography (ECC) algorithm is the best choice for support public/private key encryption because of its ability to support AES security using public keys as small as 512-bits, which is 29 times less than the traditional RSA algorithm [18]. Therefore, a hybrid cryptographic system with a combination of public/private key encryption algorithm (ECC) is the choice for mobile/wireless devices [18].

\section{G. Electromagnetic Compatibility}

Electromagnetic compatibility (EMC) is the ability of electronic devices to co-exist without adversely affecting each other's performance [29]. It has been a concern that devices connected through wireless network may interfere with life saving medical equipment [28] due to electromagnetic interference (EMI) caused by conducted or radiated electromagnetic energy from these devices. The strategy for safeguarding any possible incidents caused by EMI is to implement an effective EMC program [30].

\section{THE FUTURE FOR M-HEALTH}

Improving patient safety, reducing cost, complying with clinician's workflow and reducing time-taken for accessing and updating relevant information are the major driving forces for the development of m-health. The future m-health solutions will include increased use of prompted and interactive speech, integrated with dictation [20]. There is a trend for the convergence of devices that facilitates both touch screen and keyboard entry of data, voice dictation, voice communication, video and audio streaming. The communication network will be a mixed connectivity that incorporates wireless and wired network, Bluetooth, cell phone, 2G and 3G network, etc.

The clinicians in the future healthcare system will be the ones that carry a book-side or pocket PC size mobile device anytime, any place. The mobile device will be the record writer, referencing point and phone for oral communication. Without urgent needs, the clinicians do not really need to consult desktop computer for patient information. The whole solution is integrated into clinician's daily practice. The whole medical practice is paperless.

Some researchers projected the following trends in USA [31]:

- Within five years, virtually all clinicians will use mobile technology as a part of their work processes.

- "Next generation" information systems can run on a mobile device that has a Web browser.

- Building functionality for "smart phones," which combine PDA and cellular telephone functions, is also on vendor radar screens.

\section{CONCLUSION}

To achieve the above desirable outcomes, many challenges have to be overcome. However, healthcare workers will adopt new mobile devices and technologies if the application aligns with business objectives of the healthcare providers and mobile practice of healthcare workers.

One CIO Chuck Podesta of Berkshire Health Systems addressed the driving force for the further development of m-health like this [32]:

"We are not going to let mobile hardware stand in the way of wireless network success like it has at other organizations. It would be easier to support just a handful of devices on a wireless network. But you can't focus on what's easiest for your I.T. department--you have to focus on what's best for the end users. ".

\section{REFERENCES}

[1] Mobile Healthcare Alliance, "What is Mobile Health?", 2002, [online] Available at URL: http://www.mohca.com/faq.php3. [Accessed March 20, 2004].

[2] G. H. Malkary and A. Farrell, "Mobile nursing computing solutions: Why the resistance to adopt?', Presentation at 2005 Annual HIMSS Conference and Exhibition. February 16, 2005. Dallas Convention Center. Conference CD Recording No. 201.

[3] H. Pearson, "Doctors going wireless with souped-up PDAs", 2005, [online] http://www.msnbc.msn.com/id/710981013/ CNBC News, [Accessed April 25, 2005].

[4] M.M. Shabot, "The wireless hospital world: overcoming the fear of flying wirelessly", Presentation at 2005 Annual HIMSS 
Conference and Exhibition. February 16, 2005. Dallas Convention Center. Conference CD Recording No. 1110.

[5] M.B. Johnson, and S. Trakowski, "Setting a course toward bard code and RFID enabled wireless technologies", Presentation at 2005 Annual HIMSS Conference and Exhibition. February 14, 2005. Dallas Convention Center. Conference CD Recording No. 180.

[6] Daou Systems, Inc., "mHealth. Well-grounded, you'll fly", 2005, http://www.daou.com/emerging/mhealth.asp [Accessed April 3, 2005].

[7] Global Information. Inc., "Mobile healthcare mobile and wireless strategies for future healthcare provisioning”, 2004, [online] Available at URI

http://www.the-infoshop.com/study/ba19795_mobile_healthcare. html [Accessed April 1, 2005].

[8] Fox News Story, "Wireless access", 2004, [online] http://www.cdh.org/. [Accessed April 6, 2005].

[9] Mobile Health Data, " Government and Industry - Survey: Future is not PDAs", 2005, [online] Available at URL: http://www.mobilehealthdata.com/article.cfm?articleid=1274. [Accessed March 20, 2005].

[10] J. Thompson,"Can you read me now? WiFi and cellular delivery of the HER", Presentation at 2005 Annual HIMSS Conference and Exhibition. February 14, 2005. Dallas Convention Center. Conference CD Recording No. 131.

[11] Spyglass Consulting Group, "Mobile Computing for Physicians", 2005, [online] Available at URL:

http://www.spyglass-consulting.com/spyglass_whitepaper_doctor .html. [Accessed April 27, 2005].

[12] G. Gillespie, "The Ascent of Wireless Networks", 2004, [online] Available at URL:

http://www.mobilehealthdata.com/article.cfm?articleId=804\&ban ner=b1 [Accessed March 13, 2004].

[13] R.R. Rogoski, "Wireless by design. health management technology", 2005, [Online]. Available at URL: http://www.healthmgttech.com/cgi-bin/arttop.asp?Page $=0105 / 01$ 05wireless.htm. [Accessed March 20, 2005]

[14] P.W. Brient, "Maximizing the gains of mobility", Health Management Technology, April 2005. [Online] Available at URL: http://www.healthmgttech.com. [Accessed March 22, 2005].

[15] Mobileinfo, "Introduction to mobile health care", 2003, [online]. Available at URL:

http://www.mobileinfo.com/Applications_Vertical/Healthcare_A pplications/index.htm. [Accessed October 29, 2003].

[16] HIMSS, "Exploring barriers to nurse mobile computing", 2005, [online].http://www.healthdatamanagement.com/html/supplemen ts/himss2005/HimssNewsStory.cfm?DID=13334. [Accessed March 20, 2005].

[17] K. Anand, "CriticalApps: Wireless LAN technology", Presentation at 2005 Annual HIMSS Conference and Exhibition. February 17, 2005, Dallas Convention Center. Conference CD Recording No. 251. [Accessed March 23, 2005].

[18] J. Sutherland, "PatientKeeper platform extends the CCOW standard to mobile computing", 2003, [online] Available at URL: http://www.patientkeeper.com/download/platform/TB_Extending CCOW_Standard_12 03.pdf. [Accessed April 23, 2005].

[19] J. Sutherland, and G. Madrid, "PatientKeeper® platform extends secure and auditable encryption standards to mobile computing", 2003, [Online] Available at URL:

http://www.patientkeeper.com/download/platform/WP_Encryptio n_Standards_12_30_03.pdf. [Accessed March 24, 2005].

[20] Health Data Management, "Survey: more funds for clinical I.T", 2005, [online] Available at URL:

http://www.healthdatamanagement.com/html/PortalStory.cfm?ty pe $=$ trend \&DID=12353. [Accessed Aril 10, 2005].

[21] Mobile Health Data, "In the spotlight - Survey: Interest in PDAs declining", 2005, [online] Available at URL:

http://www.mobilehealthdata.com/article.cfm?articleId=1357\&ba nner=b1. [Accessed April 8, 2005].

[22] M.L. Baker, "Options for mobile health devices fall short", eWeek Enterprise News \& Reviews, 2005, [Online] Available at URL:

http://www.eweek.com/print_article2/0,2533,a=142790,00.asp [Accessed April 4, 2005].

[23] Health Data Management, "Trends: CIOs getting vocal about iPods", 2005, [online] Available at URL: http://www.healthdatamanagement.com/html/PortalStory.cfm?ty pe $=$ trend $\& D I D=12539$. [Accessed April 14, 2005].

[24] B. Brown, "Electronic health records can be more secure, efficient than paper", Nov 2003, [online]. Available at URL:
http://www.ecm-inc.com/news/postreview/2003/November/4heal threcord.html. [Accessed March 8, 2004].

[25] P. Cardoza, "Select a Mobile Workplace Solution", 2003 , [online]. Available at URL:

http://www.ftponline.com/wss/2003_02/magazine/columns/wirel ess/default.aspx. [Accessed March 24, 2004].

[26] D. Witters, "Medical devices EMC: Safety and effectiveness aspects of wireless connected devices", 2003, [online] http://www.mohca.org/presentations/WittersEMC2.pdf. [Accessed April 16, 2005].

[27] Mobile Healthcare Alliance, "MoHCA White Paper: Management of wireless EMC in the healthcare environment", 2004, [online] Available at URL: http://www.mohca.org/presentations/MoHCAemcV9.doc[Access ed April 21, 2005].

[28] Health Data Management, "Sun Exec: RFID will Beat Bar Codes", 2005, [online] Available at URL:

http://www.healthdatamanagement.com/html/PortalStory.cfm?ty pe $=$ trend\&DID=12468. [Accessed March 25, 2005].

[29] Technovelgy, "Problems with RFID", 2002, [online] Available at URL:

http://www.technovelgy.com/ct/Technology-Article.asp?ArtNum $=20$. [Accessed April 29, 2005].

[30] Mobile Health Data, "Applied digital adds RFID maker", 2005, [online] Available at URL:

http://www.mobilehealthdata.com/article.cfm?articleId=1394\&ba nner=p6. [Accessed April 6, 2005].

[31] J. Goedert, "Mobile Clinical Apps: The Race is On: Major software vendors scramble to meet clinicians needs for mobile computing", 2003, [online]. Available at URL: http://mobilehealthdata.com/article.cfm?articleid $=4108$ [Accessed November 4, 2003].

[32] E. Giguere, "Designing Mobile Applications for the Enterprise", 2003, [online]. Available at URL:

http://www.perfectxml.com/Conf/Wrox/Files/eric1text.pdf. [Accessed October 31, 2003]. 